\title{
Effects of the crystal structure in the dynamical electron density-response of hcp transition metals
}

\author{
I. G. Gurtubay, ${ }^{(1)}$ Wei Ku, ${ }^{(2)}$ J. M. Pitarke, ${ }^{(1,3)}$ and A. G. Eguiluz ${ }^{(4,5)}$ \\ (1) Materia Kondentsatuaren Fisika Saila, Zientzi Fakultatea, Euskal Herriko \\ Unibertsitatea, 644 Posta kutxatila, 48080 Bilbo, Basque Country, Spain \\ (2) Department of Physics, Univ. of California, Davis, CA 95616-867r, USA \\ (3) Donostia International Physics Center (DIPC) and Centro Mixto \\ CSIC-UPV/EHU, Donostia, Basque Country, Spain \\ (4) Department of Physics and Astronomy, Univ. of Tennessee, Knoxville, TN \\ 37996-1200, USA \\ (5) Solid State Division, Oak Ridge National Laboratory, Oak Ridge, TN 37831-1200, \\ $U S A$
}

\begin{abstract}
We present an all-electron study of the dynamical density-response function of hexagonal close-packed transition metals Sc and Ti. We elucidate various aspects of the interplay between the crystal structure and the electron dynamics by investigating the loss function, and the associated dielectric function, for wave-vector transfers perpendicular and parallel to the hexagonal plane. As expected, but contrary to recent work, we find that the free-electron-like aspects of the dynamical response are rather isotropic for small wave vectors. The crystal local-field effects are found to have an impact on the plasmon energy for small wave vectors, which gives rise to an interplay with the exchange-correlation effects built into the many-body kernel. The loss function lineshape shows a significant dependence on propagation direction; in particular, for propagation on the hexagonal plane the plasmon hybridizes substantially with fine structure due to $d$-electron transitions, and its dispersion curve becomes difficult to establish, beyond the small wave vector limit. The response is calculated in the framework of time-dependent density functional theory (TDDFT), based on a full-potential linearized augmented-plane-wave (LAPW) ground-state, in which the exchange-correlation effects are treated in the local-density approximation.
\end{abstract}

Electronic excitations in solids can be studied from the knowledge of the dynamical density-response function, which is directly related to observables such as inelastic scattering cross sections for fast electrons and hard x-rays.

For many years, theoretical investigations of valence-electron excitations were carried out on the basis of the free-electron gas (FEG) model (or jellium model), in which valence electrons are described by an assembly of interacting electrons embedded in a uniform background of positive charge. The physics of the FEG model is completely controlled by only one parameter, the valence-electron density, $n_{0}$ or, equivalently, by the electron-density parameter $r_{s}$ which is related to $n_{0}$ through $1 / n_{0}=4 \pi / 3\left(r_{s} a_{0}\right)^{3}$, $a_{0}$ being the Bohr radius. As we shall illustrate below, in real materials the physics of the excitations is more complex; indeed, the loss spectra shows qualitative departures from the FEG description.

In the past few years, ab initio calculations of the density-response function of many simple [1]-[7] and noble [8]-[10] metals have been reported. Interestingly, in a number 
of cases it has been concluded that remarkable anomalies revealed by experimental measurements arise mainly from band-structure effects [11]. Examples include the fine structure in the inelastic X-ray scattering data for aluminum [1], the negative plasmon energy dispersion of cesium [3],[5], and the positive dispersion of the linewidth of the plasmon in potassium [6] .

In this paper we utilize an all-electron method to compute the density-response function of Sc and Ti starting from the knowledge of an LAPW ground state [6],[7],[12]. Our presentation centers on a discussion of the dominant feature of the excitation spectrum for these hexagonal closed-packed transition metals for relatively small wave vectors - the collective, plasmon-like mode, and its interplay with the effects of the crystal lattice; in particular, we assess the impact of the so-called crystal local fields, and the dependence on propagation direction. We conclude that the crystal local-field effects are not negligible, in the small wave vector limit; this brings about an interplay with the effects of exchange and correlation, which thus impact the plasmon dispersion for small wave vectors, unlike the case of the FEG. Similarly, the dependence on propagation direction of the response function is rather significant: for wave vector transfers along the hexagonal plane, the plasmon hybidizes with the fine structure caused by $d$-electron transitions and its dispersion curve becomes difficult to establish beyond the small wave vector limit. Comparison is made with the recent pseudopotential-based calculations of the density-response function of Sc by Schöne and Ekardt [13]; the surprising result reported by these authors that the plasmon-like excitation is not realized for small wave vectors for propagation in the hexagonal plane is not supported by our calculations. Our results agree with the intuitive expectation that the free-electron-like response is not affected drastically by the propagation direction.

\section{Theory}

The density-response function $\chi\left(\mathbf{r}, \mathbf{r}^{\prime} ; \omega\right)[14]$ of an interacting electron system gives, within linear-response theory, the electron density induced in the system when the latter is exposed to an external potential $V^{e x t}(\mathbf{r}, \omega)$ through the equation

$$
\rho^{i n d}(\mathbf{r}, \omega)=\int \mathrm{d} \mathbf{r}^{\prime} \chi\left(\mathbf{r}, \mathbf{r}^{\prime} ; \omega\right) V^{e x t}\left(\mathbf{r}^{\prime}, \omega\right) .
$$

In the framework of time-dependent density-functional theory (TDDFT) [15], the exact density-reponse function $\chi\left(\mathbf{r}, \mathbf{r}^{\prime} ; \omega\right)$ obeys the integral equation [16]

$$
\begin{aligned}
\chi\left(\mathbf{r}, \mathbf{r}^{\prime} ; \omega\right) & =\chi^{S}\left(\mathbf{r}, \mathbf{r}^{\prime} ; \omega\right)+\int \mathrm{d} \mathbf{r}_{1} \int \mathrm{d} \mathbf{r}_{2} \chi^{S}\left(\mathbf{r}, \mathbf{r}_{1} ; \omega\right) \\
\times & {\left[v\left(\mathbf{r}_{1}-\mathbf{r}_{2}\right)+f_{x c}\left(\mathbf{r}_{1}, \mathbf{r}_{2} ; \omega\right)\right] \chi\left(\mathbf{r}_{2}, \mathbf{r}^{\prime} ; \omega\right), }
\end{aligned}
$$

where $\chi^{S}\left(\mathbf{r}, \mathbf{r}^{\prime} ; \omega\right)$ is the single-particle density-response function of the unperturbed Kohn-Sham (KS) system [17], $v\left(\mathbf{r}-\mathbf{r}^{\prime}\right)$ is the bare Coulomb interaction, and $f_{x c}\left(\mathbf{r}, \mathbf{r}^{\prime} ; \omega\right)$ accounts for all dynamical exchange-correlation (xc) effects.

For a periodic crystal it is convenient to work in Fourier space, so we write

$$
\chi\left(\mathbf{r}, \mathbf{r}^{\prime}, \omega\right)=\frac{1}{\Omega} \sum_{\mathbf{k}}^{B Z} \sum_{\mathbf{G}, \mathbf{G}^{\prime}} \mathrm{e}^{\mathrm{i}(\mathbf{k}+\mathbf{G}) \cdot \mathbf{r}} \mathrm{e}^{-\mathrm{i}\left(\mathbf{k}+\mathbf{G}^{\prime}\right) \cdot \mathbf{r}^{\prime}} \chi_{\mathbf{G}, \mathbf{G}^{\prime}}(\mathbf{q}, \omega)
$$


with a similar Fourier expansion for $\chi^{S}\left(\mathbf{r}, \mathbf{r}^{\prime} ; \omega\right)$. The Fourier coefficients of the KS response function, $\chi_{\mathbf{G}, \mathbf{G}^{\prime}}^{S}(\mathbf{k}, \omega)$, can be written as

$$
\begin{aligned}
& \chi_{\mathbf{G}, \mathbf{G}^{\prime}}^{S}(\mathbf{k}, \omega)=\frac{1}{\Omega} \sum_{\mathbf{k}^{\prime}}^{B Z} \sum_{n, n^{\prime}} \frac{f_{\mathbf{k}^{\prime}, n}-f_{\mathbf{k}^{\prime}+\mathbf{k}, n^{\prime}}}{E_{\mathbf{k}^{\prime}, n}-E_{\mathbf{k}^{\prime}+\mathbf{k}, n^{\prime}}+\hbar(\omega+\mathrm{i} \eta)} \\
& \quad \times\left\langle\phi_{\mathbf{k}^{\prime}, n}\left|e^{-\mathrm{i}(\mathbf{k}+\mathbf{G}) \cdot \mathbf{r}}\right| \phi_{\mathbf{k}^{\prime}+\mathbf{k}, n^{\prime}}\right\rangle\left\langle\phi_{\mathbf{k}^{\prime}+\mathbf{k}, n^{\prime}}\left|e^{\mathrm{i}\left(\mathbf{k}+\mathbf{G}^{\prime}\right) \cdot \mathbf{r}}\right| \phi_{\mathbf{k}^{\prime}, n}\right\rangle,
\end{aligned}
$$

where $\Omega$ represents the normalization volume, $\mathbf{G}$ and $\mathbf{G}^{\prime}$ are vectors of the reciprocal lattice, and the second sum runs over the band structure for each wave vector $\mathbf{k}^{\prime}$ of the first Brilloun Zone (BZ). $\phi_{\mathbf{k}, n}(\mathbf{r})$ and $E_{\mathbf{k}, n}$ are Bloch eigenfunctions and eigenvalues of the KS Hamiltonian of ground-state density-functional theory (DFT) [17], and $f_{\mathbf{k}, n}$ are the occupation numbers, with due account for spin degeneracy.

In the above Fourier representation, eq. (2) turns into a matrix equation

$$
\begin{aligned}
& \chi_{\mathbf{G}, \mathbf{G}^{\prime}}(\mathbf{k}, \omega)=\chi_{\mathbf{G}, \mathbf{G}^{\prime}}^{S}(\mathbf{k}, \omega)+\sum_{\mathbf{G}^{\prime \prime}} \sum_{\mathbf{G}^{\prime \prime \prime}} \chi_{\mathbf{G}, \mathbf{G}^{\prime \prime}}^{S}(\mathbf{k}, \omega) \\
& \times\left[v_{\mathbf{G}^{\prime \prime}}(\mathbf{k}) \delta_{\mathbf{G}^{\prime \prime}, \mathbf{G}^{\prime \prime \prime}}+f_{\mathbf{G}^{\prime \prime}, \mathbf{G}^{\prime \prime \prime}}^{x c}(\mathbf{k}, \omega)\right] \chi_{\mathbf{G}^{\prime \prime \prime}, \mathbf{G}^{\prime}}(\mathbf{k}, \omega),
\end{aligned}
$$

which we solve numerically. The size of this matrix equation is a measure of the importance of the crystal local-field effects, arising from the inhomogeneity of the electronic environment in the periodic crystal potential. For later reference, we note that ignoring the crystal local-field effects corresponds to solving eq. (5) while keeping only the zero-zero element of the KS response matrix.

Within the first Born approximation the inelastic scattering cross-section for hard $\mathrm{x}$-rays and fast electrons corresponding to wave-vector transfer $\mathbf{q}=\mathbf{k}+\mathbf{G}$, is proportional to the dynamic-structure factor

$$
S(\mathbf{q}, \omega)=-2 \hbar \Omega \frac{1}{v_{\mathbf{G}}(\mathbf{k})} \operatorname{Im}\left[\epsilon_{\mathbf{G}, \mathbf{G}}^{-1}(\mathbf{k}, \omega)\right],
$$

where $\operatorname{Im}\left[\epsilon_{\mathbf{G}^{-}, \mathbf{G}^{\prime}}^{-1}(\mathbf{k}, \omega)\right]$ is the so-called energy-loss function and is related to the response function through

$$
\epsilon_{\mathbf{G}, \mathbf{G}^{\prime}}^{-1}(\mathbf{k}, \omega)=\delta_{\mathbf{G}, \mathbf{G}^{\prime}}+v_{\mathbf{G}}(\mathbf{k}) \chi_{\mathbf{G}, \mathbf{G}^{\prime}}(\mathbf{k}, \omega) .
$$

The plasmon-energy dispersion is given by the frequencies at which the real part of the dielectric function, $\epsilon(\mathbf{k}, \omega)$, is close to zero and the imaginary part is small. $\operatorname{Im} \epsilon^{-1}(\mathbf{k}, \omega)$ is therefore a maximum. Throughout this work the plasmon-energy is taken to be the energy location of the main peak of the energy-loss function.

\section{Numerical Implementation}

The key ingredient in the calculation of the energy-loss spectrum is the KS response function $\chi^{S}$ of eq. (4). For this purpose, we need the KS states which we obtain within the local-density approximation (LDA) [18], in terms of a variational expansion in the LAPW basis [19]:

$$
\phi_{\mathbf{k}, n}(\mathbf{r})=\frac{1}{\Omega} \sum_{\mathbf{G}} C_{n}(\mathbf{k}+\mathbf{G}) \psi_{\mathbf{k}+\mathbf{G}}^{L A P W}(\mathbf{r})
$$


The LAPW functions $\psi_{\mathbf{k}+\mathbf{G}}^{L A P W}(\mathbf{r})$ are obtained dividing the unit cell into two regions: non-overlapping atomic spheres centered at nuclear sites and the interstitial region between the spheres. This allows a faithful description of the localized strong oscillations near the atomic site, where atomic-like functions are used, and the smooth behaviour of the interstitial region, where plane waves are employed. Local orbitals are also introduced in order to have an accurate description of the semi-core states.

The use of the symmetry properties of the crystal allows us to work with Bloch states that involve momentum transfers in the irreducible Brillouin Zone (IBZ) instead of those in the whole zone. If $\tilde{\mathbf{k}}$ is a vector of the IBZ,

$$
\phi_{\mathbf{k}, n}(\mathbf{r})=\phi_{\mathrm{R}^{-1} \tilde{\mathbf{k}}, n}(\mathbf{r})=e^{-\mathrm{i} \tilde{\mathbf{k}} \cdot \boldsymbol{\tau}(\mathrm{R})} \phi_{\tilde{\mathbf{k}}, n}(\mathrm{R} \cdot \mathbf{r}+\boldsymbol{\tau}),
$$

where $\mathrm{R}(\tilde{\mathbf{k}})$ runs over the sub-set of the point group of the crystal that generates the star of $\tilde{\mathbf{k}}$ and $\boldsymbol{\tau}(\mathrm{R})$ is the partial lattice displacement corresponding to the space-group operation $\{\mathrm{R}, \boldsymbol{\tau}(\mathrm{R})\}$.

The evaluation of the matrix elements in eq. (4) is performed with a code that runs in parallel and scales linearly with the number of nodes used. With the matrix elements at hand we perform the sum over k-points and over the pairs of bands allowed by the occupation numbers. We solve equation (5) using two different approximations for $f_{x c}$. In the random-phase approximation (RPA), $f_{x c}=0$. In the so-called adiabatic local-density approximation (ALDA),

$$
f_{\mathrm{xc}}^{\mathrm{ALDA}}\left(\mathbf{r}, \mathbf{r}^{\prime} ; \omega\right)=\delta\left(\mathbf{r}-\mathbf{r}^{\prime}\right)\left[\frac{\mathrm{d}^{2} E_{\mathrm{xc}}(n)}{\mathrm{d} n^{2}}\right]_{n=n_{0}(\mathbf{r})},
$$

where $E_{\mathrm{xc}}(n)$ is the exchange-correlation energy of a uniform electron gas of density $n$, and $n_{0}(\mathbf{r})$ is the ground-state density.

We apply the above formalism to hexagonal closed-packed transition metals Sc and $\mathrm{Ti}$, whose electronic configurations are $[\mathrm{Ar}] 3 d^{1} 4 s^{2}$ and $[\mathrm{Ar}] 3 d^{2} 4 s^{2}$, respectively. The lattice parameters used are $a=2.95 \AA$ and $c=4.68 \AA$ for Ti and $a=3.31 \AA$ and $c=5.28 \AA$ for Sc [20]. The ground state is obtained with a cut-off parameter $R_{M T} K_{\max }=8$. Inside the atomic spheres, the LAPW wave functions are expanded in spherical harmonics $Y_{l m}$ up to $l=10$. The response function was evaluated for $\mathbf{q}$ wave vectors perpendicular and parallel to the hexagonal plane, using $8 \times 8 \times 16$ and $16 \times 16 \times 8$ BZ samplings (the corresponding number of points in the IBZ being 90 and 150, respectively) and keeping KS states up to 7.5Ry, thereby ensuring convergence in the energy range under study. The $3 s$ and $3 p$ states were treated as semi-core states.

\section{Results}

The upper left (right) panel in Fig. 1 shows the dynamical-structure factor obtained within ALDA for $\mathrm{Ti}(\mathrm{Sc})$ for energies up to the onset of semicore excitations $(\sim 32.5$ $\mathrm{eV}$ in $\mathrm{Ti}$ and $\sim 27.7 \mathrm{eV}$ in $\mathrm{Sc}$ ) along the (001) direction. We have inverted eq. (5) using up to 9 shells (55 G-vectors) [21]. For clarity, only results for the scalar inversion, corresponding to a $1 \mathrm{x} 1$ matrix calculation with the $\mathbf{G}=\mathbf{G}^{\prime}=0$ element (dashed line), and for matrix inversion using 11 (dotted-line), 23 (solid line) and 35 (open circles) G-vectors are displayed. It's apparent that convergence is reached for the energy 

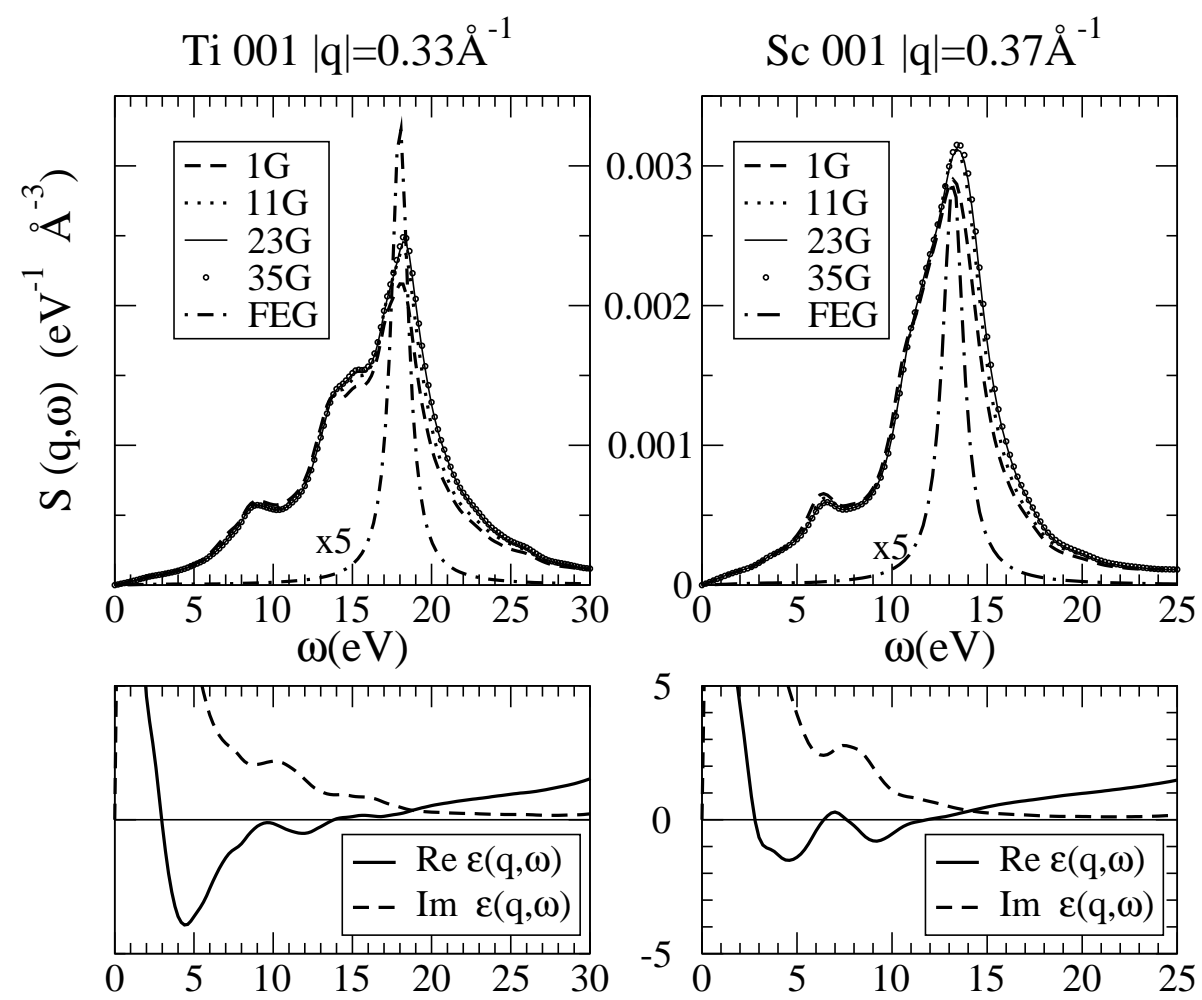

Figure 1: Top panel: impact of local-filed effects on the dynamic structure function of $\mathrm{Ti}$ and Sc along the hexagonal axis within ALDA. The values of the momentum transfer are $\mathbf{q}=4 / 16(001)$ and $\mathbf{q}=5 / 16(001)$ respectively, both in units of $2 \pi / c$. The dotted-dashed line corresponds to a FEG calculation with $r_{s}=1.92$ a.u. (Ti) and $r_{s}=2.38$ a.u. (Sc). Lower panel: dielectric function.

range under study (the physics of the dynamical response above the M-edge will be the subject of another publication); the results shown in Figs. 2 and 3 correspond to the converged $35 \mathbf{G}$-vector calculations [22]. $S(\mathbf{q}, \omega)$ displays a broad plasmon-like peak, whose position is shifted upwards (between $0.5 \mathrm{eV}$ and $1.0 \mathrm{eV}$ ) by the crystal local-field effects. These peaks correspond (see lower panels of Fig. 1) to zero values of $\operatorname{Re} \epsilon(\mathbf{q}, \omega)$ where $\operatorname{Im} \epsilon(\mathbf{q}, \omega)$ is small.

For comparison, the upper panels of Fig. 1 also show (dotted-dashed line) the dynamical structure factor calculated within the FEG model for $r_{s}=1.92$ a.u. (Ti) and $r_{s}=2.38$ a.u. (Sc) for the same wave-vector as the ab initio calculation, $r_{s}$ being the electron density equivalent to that of valence electrons, $3 d^{1} 4 s^{2}$ and $3 d^{2} 4 s^{2}$, in $\mathrm{Ti}$ and Sc respectively [23]. Clearly, as anticipated at the outset, the overall loss functions of these transition metals bear little resemblance to the FEG model, other than the presence of a broad collective mode; again, the essentially overdamped mode which defines the leading feature in Fig. 1 corresponds to a dielectric function whose nature is quite removed from the simple, clean, Drude-like zero which defines the textbook plasmon. Additional peaks around $9 \mathrm{eV}$ in $\mathrm{Ti}$ and around $6 \mathrm{eV}$ in Sc arise from transitions involving the $d$-electrons and will be discussed elsewhere.

In order to investigate the dependence on the direction of the momentum transfer, we show in Fig. 2 the ALDA dynamic structure factor $\mathrm{S}(\mathbf{q}, \omega)$ for $\mathrm{Ti}$ and Sc along the (001) and (110) directions for $q$ values of the same order of magnitude. Dashed 


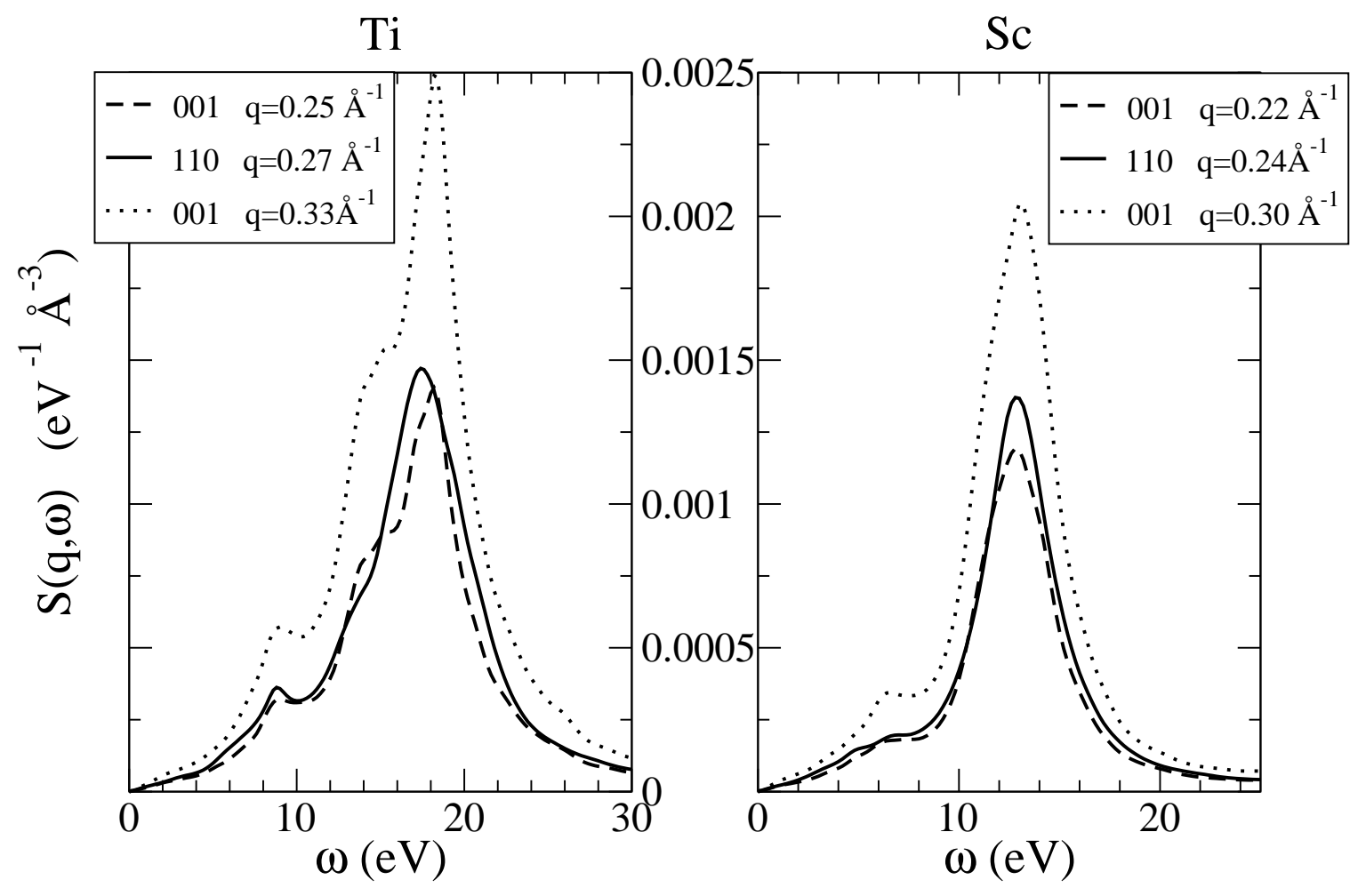

Figure 2: Comparison of the dynamical structure factor for similar values of the momentum transfer perpendicular (001) and parallel to the hexagonal plane (110) for Ti (left) and Sc (right) within ALDA.

and dotted lines represent $S(\mathbf{q}, \omega)$ for $\mathbf{q}=3 / 16(001)$ and $\mathbf{q}=4 / 16(001)$, respectively, in units of $2 \pi / c$. The calculation along the (110) direction (solid line) is for $\mathbf{q}=1 / 16(110)$, in units of $2 \pi / a$. The curves along the (001) direction are calculated with a $8 \times 8 \times 16$ sampling over the BZ and the ones in the (110) direction with a $16 \times 16 \times 8$ sampling [24]. As the left panel on Fig. 2 illustrates, for both propagation directions the plasmon peak in Ti hybridizes with fine structure due to $d$-electron transitions (prominent peak and shoulder at its left, respectively). This hybridization is more pronounced along the hexagonal plane. For this reason, the identification of a plasmon peak for larger momentum transfers along this direction becomes very ambiguous.

In recent work [13], Schöne and Ekardt have reported pseudopotential-based calculations of the dynamic structure factor for Sc. The intriguing aspect of their results is that they suggest that the collective mode is not realized for small wave vectors for propagation along the hexagonal plane. By contrast, as shown in Fig. 2 the nature of the collective-like response in $\mathrm{Sc}$ (and also in $\mathrm{Ti}$ ) is, for small wave vectors, quite similar for propagation directions parallel and perpendicular to the hexagonal plane. Our result agrees with intuitive expectations; on that basis we submit that it is correct.

Fig. 3 shows the plasmon-energy dispersion for both directions within RPA (solid symbols) and ALDA (empty symbols). Circles and squares correspond to wave-vector transfers that are perpendicular and parallel to the hexagonal plane. As expected, the ALDA plasmon energy shifts to slightly smaller values compared to that of the RPA, due to a less effective electron-electron interaction.

An interesting feature in both panels of Fig. 3 is that, in the small wave vector 

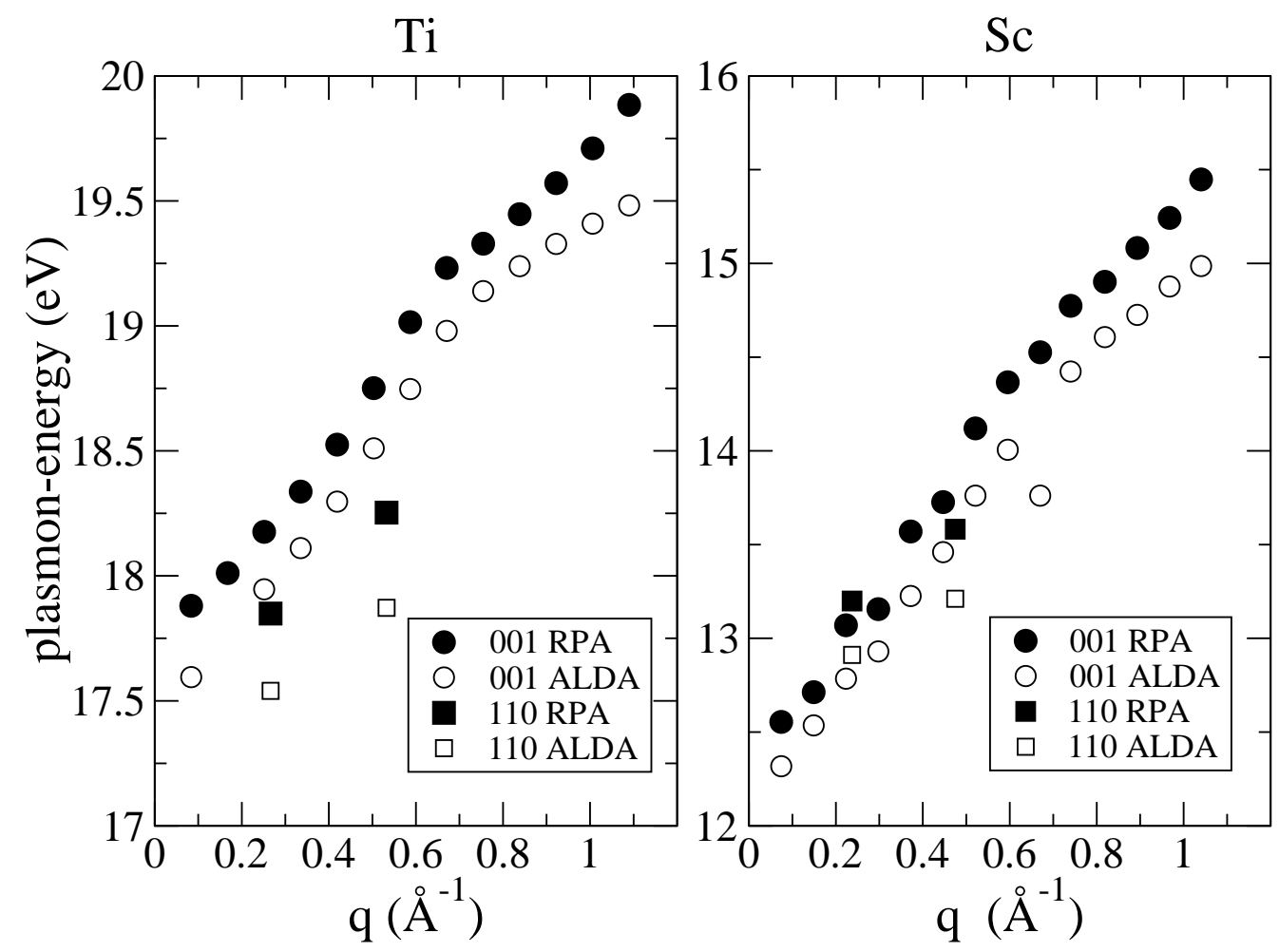

Figure 3: Plasmon-energy dispersion for Ti (left) and Sc (right) along the 001 direction (circles) and along the 110 direction (squares) within RPA (solid symbols) and within ALDA (empty symbols).

limit, RPA and ALDA do not yield the same result, as it occurs in the FEG. Indeed, in the case of the FEG, the Coulomb term dominates over the exchange-correlation contribution when $\mathbf{q} \rightarrow 0$. In the case of the $a b$ initio calculation, the difference between RPA and ALDA arises from the inclusion of crystal local-field effects and its interplay with the exchange correlation effects: for small wave vectors, $\mathbf{q}$ in eq. (6) lies in the BZ and hence, the element we consider after solving the matrix equation (5) for $\chi_{\mathbf{G}, \mathbf{G}^{\prime}}$ is the $\mathbf{G}=\mathbf{G}^{\prime}=0$ element; thus, in this case $\mathbf{k}=\mathbf{q}$.

Through the inversion process there is a "mixing" between large wave vector arguments $\mathbf{k}+\mathbf{G}$ in $\chi^{S}$ and the small wave vector $\mathbf{q}$ of the plasmon, causing a shift of the collective peak to larger energies. On the other hand, as mentioned before, exchangecorrelation effects cause a downwards shift, smaller than the former one, provoking thus the difference [25]. The partial cancellation between both effects leads to a net difference between the converged, ALDA result, and the scalar RPA result, for the small-q plasmon dispersion curve.

$\mathrm{Ti}$ and Sc show positive plasmon dispersions and disperse faster along the hexagonal axis. For $|\mathbf{q}|$ values larger than $\sim 0.5 \AA^{-1}$, the assignment of a collective peak in the (110) direction becomes a difficult task. By contrast, this assignment is still possible along the (001) direction. This fact ratifies, once more, the importance of the underlying crystal structure.

We note that for $\mathrm{Ti}$, the plasmon energy $(\sim 19 \mathrm{eV})$ is in good agreement with the one measured in reference [26] for $q=0.7 \AA^{-1}$ along (001). 


\section{Conclusions}

We have presented results of calculations of the dynamic response function of hcp Sc and Ti based on a full-potential linearized augmented plane wave method. We have found that crystal local-field effects have a non-negligible impact on the plasmon energy for small wave vectors causing an interplay between the crystal potential and the exchange-correlation effects. We have investigated the dependence on propagation direction of the dynamical-structure factor and we have shown that, contrary to a recent report [13], the plasmon loss is quite isotropic; however, the lineshape of the energy loss shows considerable dependence on the direction of the wave vector transfer: along the hexagonal plane, the identification of a dispersion relation for the plasmon energy beyond the small wave vector limit, becomes more difficult due to hybridization with the fine structure originated by $d$-electron transitions.

I. G. G. acknowlegdes support by the Basque Government, through the Hezkuntza, Unibertsitate eta Ikerketa Saila. I. G. G. is grateful to the hospitality of the University of Tennessee, Knoxville and the Solid State Division at Oak Ridge National Labora-

tory, where most of this work has been carried out. A. G. E. acknowledges support from NSF ITR DMR-0219332.

\section{References}

[1] A. A. Quong and A. G. Eguiluz, Phys. Rev. Lett. 70, 3955 (1993); N. E. Maddocks, R. W. Godby, and R. J. Needs, Europhys. Lett., 27, 681 (1994); A. Fleszar, A. A. Quong, and A. G. Eguiluz, Phys. Rev. Lett. 74, 590 (1995).

[2] N. E. Maddocks, R. W. Godby, and R. J. Needs, Phys. Rev. B 49, 8502 (1994).

[3] F. Aryasetiawan and K. Karlsson, Phys. Rev. Lett. 73, 1679 (1994).

[4] K. Karlsson and F. Aryasetiawan, Phys. Rev. B 52, 4823 (1995).

[5] A. Fleszar, R. Stumpf, A. G. Eguiluz, Phys. Rev. B 55, 2068 (1997).

[6] Wei Ku and A. G. Eguiluz, Phys. Rev. Lett. 82, 2350 (1999).

[7] A. G. Eguiluz, W. Ku and J. M. Sullivan. J. Phys. Chem. Solids, 61 , 383 (2000).

[8] I. Campillo, A. Rubio and J. M. Pitarke, Phys. Rev. B 59, 12188 (1999).

[9] M. A. Cazalilla, J. S. Dolado, A. Rubio, and P. M. Echenique, Phys. Rev. B 61, 8033 (2000).

[10] I. G. Gurtubay, J. M. Pitarke, I. Campillo and A. Rubio, Comp. Mater. Sci. 22, $123(2001)$.

[11] See for example Ref. [6] or Ref. [2] .

[12] W. Ku and A. G. Eguiluz, preprint.

[13] W. D. Schöne and W. Eckardt, J. Phys.:Condens. Mater 14,4669 (2002). 
[14] P. Nozieres and D. Pines, The Theory of Quantum Liquids, Benjamin, New York, 1966.

[15] E. Runge, E. K. U. Gross, Phys. Rev. Lett. 52, 997 (1984).

[16] M. Petersilka, U. J. Gossmann, and E. K. U. Gross, Phys. Rev. Lett. 76, 1212 (1996).

[17] P. Hohenberg and W. Kohn, Phys. Rev. 136, B864 (1964); W. Kohn and L. Sham, Phys. Rev. 140, A1133 (1965).

[18] We use the Perdew-Wang parametrization of the exchange-correlation energy of a uniform electron gas. (J. P. Perdew, Y. Wang, Phys. Rev. B 45, 13244 (1992).)

[19] P. Blaha, et al. WIEN97.8, Technical University of Vienna, 1997. D. J. Singh, Plane waves, Pseudopotentials, and the LAPW method, Kluwer, Boston, 1994.

[20] N. W. Aschroft eta N. D. Mermin, Solid State Physics, Saunders College Publishing, 1976.

[21] The first 9 shells for an hcp crystal contain 1, 3, 9, 11, 23, 35, 37, 43 and 55 G-vectors.

[22] The numerical damping introduced in eq. (4), $\eta$, has been set equal to $0.65 \mathrm{eV}$. Smaller values of $\eta$ sharpen the structures of the energy-loss function without adding new information.

[23] The numerical broadening used is also $0.65 \mathrm{eV}$ and is responsible for the finite linewidth of the FEG peak.

[24] This choice of the samplings over the BZ for each direction allows us to use the same value for $\eta$ and therefore the comparison of the lineshapes in both directions is meaningful.

[25] See ref. [5] for a similar discussion.

[26] A. T. Macrander, P. A. Montano, D. L. Price, V. I. Kushnir, R. C. Blasdell C. C. Kao and B. R. Cooper, Phys. Rev. B 54, 305 (1996). 